\title{
Performance evaluation of multi-sensor data-fusion systems in launch vehicles
}

\author{
B N SURESH and K SIVAN \\ Avionics Entity, Vikram Sarabhai Space Centre, Thiruvananthapuram 695 022, \\ India \\ e-mail: bn_suresh@vssc.org
}

\begin{abstract}
In this paper, the utilization of multi-sensors of different types, their characteristics, and their data-fusion in launch vehicles to achieve the goal of injecting the satellite into a precise orbit is explained. Performance requirements of sensors and their redundancy management in a typical launch vehicle are also included. The role of an integrated system level-test bed for evaluating multi-sensors and mission performance in a typical launch vehicle mission is described. Some of the typical simulation results to evaluate the effect of the sensors on the overall system are highlighted.
\end{abstract}

Keywords. Multi-sensor data-fusion systems; launch vehicles; satellite injection; redundancy management.

\section{Introduction}

The launch vehicle mission is to inject a specified satellite into the required orbit within the specified accuracies. Deviations in the orbit achieved by the launch vehicle, need to be corrected at the expence of satellite fuel, which in turn reduces the useful payload or reduces satellite lifetime in the mission-defined final orbit. Since the cost involved in each satellite launch is large, the success of the launch vehicle mission is rated against the accuracy with which the satellite is injected.

The launch vehicle is a complex system, which contains large numbers of subsystems with different functions. In order to achieve the mission-defined target conditions precisely without violating vehicle safety limits, all these subsystems need to perform as planned. Being an autonomous system, each subsystem is provided with sensors to measure and control performance within the specified limits (Sivan et al 2000).

The sensors used in the launch vehicle are categorized into four types (Sivan et al 2000; Chidambaram et al 1996; Puri 2002). Functionally, they are used for performance monitoring, subsystem functions, and optimum propulsion performance and for the functions of navigation, guidance and control (NGC). The propulsion subsystems have to provide the necessary energy to reach the target, ensure engine safety and optimise propellant management. The NGC subsystem is the brain of a launch vehicle, which directs energy in an optimal direction to achieve the target accurately even under a dispersed flight environment. Hence the sensors used for propulsion control and NGC functions are very crucial for mission success and vehicle safety. 
In the integrated NGC-propulsion control (NGC-PC) system, sensor data-fusion is carried out in two ways: the same sensor data used for several functions and different sensor data used for a single function. Based on the functional requirements of each subsystem, different sensors with different characteristics are used. Thus, the launch vehicle is a multi-sensor fusion system, wherein sensor data of different types and characteristics are used in different subsystems for the single goal of precisely injecting the satellite into the mission-defined orbit.

The characteristics of different sensors are selected so as to meet the subsystem requirements, to avoid interactions between subsystems and to achieve the mission accuracy (Puri 2002). The location of each sensor is also an important factor. Proper selection of each sensor characteristic is crucial for vehicle safety, mission reliability and accuracy.

Wherever mission critical sensors are involved, sensor redundancy is built-in. Appropriate failure detection and isolation (FDI) algorithms are implemented to isolate degraded and failed sensors. Even in the case of failure of all sensors, mission salvage options are incorporated (Puri 2002).

Sensors are validated at subsystem level and integrated level tests using appropriate test beds. In subsystem level tests, sensor characteristics are evaluated against specifications (Shukkoor et al 2000; Gupta \& Sajeendran 2002). If sensor performance is not achieved, appropriate corrections are incorporated to improve performance. In integrated level tests, the effects of sensor characteristics on mission performance, sensor performance in integrated flight configuration in the presence of other subsystems, and interaction between subsystems and end-to-end signal flow are evaluated (Sivan et al 2000).

This paper addresses the multi-sensor environment for the effective management of the integrated NGC-propulsion control (NGC-PC) systems on launch vehicles. The types of sensors used in launch vehicles, their characteristics, their data-fusion and mission performance evaluation, using these sensors are described. Details of the NGC-PC system along with sensors for a typical launch vehicle are explained and the characteristics of each sensor/the criteria for selecting the same are included. Sensor redundancy, FDI logic and mission salvage mode options are elaborated along with the sensor and mission performance evaluation tests.

\section{Integrated NGC-PC system and sensors}

The functions of integrated NGC-PC systems are:

- To ensure optimum propulsion performance and propellant management.

- To achieve mission accuracy even under dispersed flight environment.

- To ensure vehicle safety.

The integrated NGC-PC system of a typical launch vehicle is given in figure 1 . The sensors used for carrying out integrated NGC-PC functions are:

- Accelerometers for measuring vehicle acceleration along the body axes.

- Gyros for measuring vehicle rates about the body axes.

These sensors are located at the vehicle equipment bay (VEB).

- Rate gyro packages for measuring rates about the body axes.

These sensors are located at the vehicle inter stage (IS).

- Propellant flow rate sensors for measuring propellant flow rates.

- Temperature sensors for measuring propellant temperature.

These sensors are located at the engine bay (EB). 
$\mathrm{EB}$

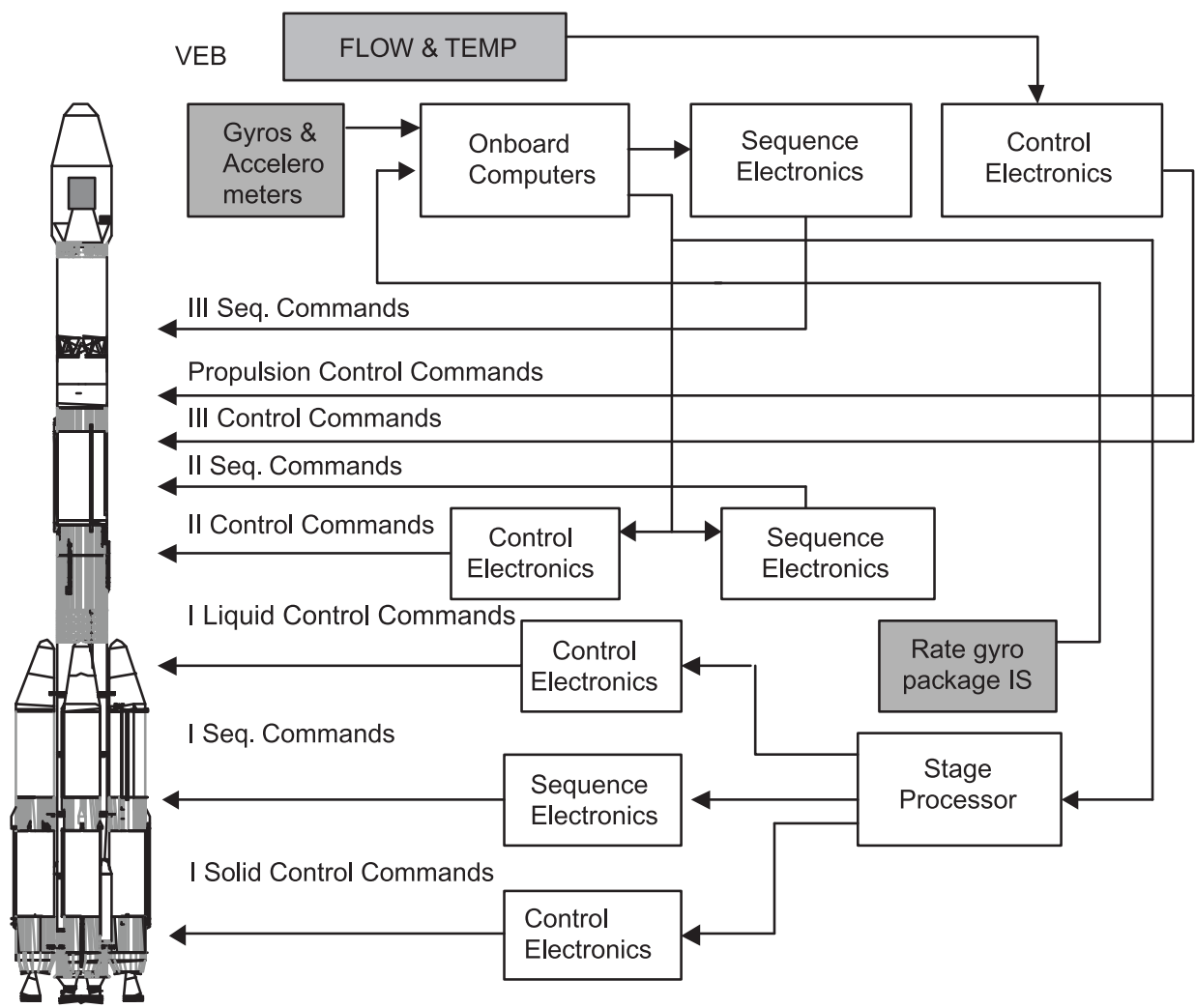

Figure 1. Integrated NGC-PC system.

\subsection{NGC functions}

Misalignments of different systems with respect to the integrated vehicle, and disturbances (both external and internal to the vehicle) and dispersions of the vehicle subsystem performance during flight cause deviation of the vehicle attitude from that desired, and thus the actual trajectory gets deviated from the intended path. The onboard NGC system senses these deviations during flight and carries out corrective action in real time so as to achieve the mission target even under the dispersed flight environment.

The navigation software loaded in the onboard computer uses the accelerometer data and rate sensed by the dry tuned gyro. From the sensor data, the processor computes the vehicle instantaneous attitude, position and velocity vectors with respect to an inertial frame. In addition to these, the processed attitude rate and acceleration information are communicated to other elements of the NGC system.

Closed loop guidance, digital autopilot and vehicle sequencing functions are also carried out using onboard computers. Using the navigation data, the guidance software computes the desired attitude of the vehicle in order to reach the targeted orbit by the optimum path. The autopilot software uses the attitude computed by navigation software along with processed rates of dry-tuned gyros and rate gyros. Using these data, the autopilot computes the necessary control commands to achieve the desired attitude as required by the guidance software. Using 
various flags computed by the guidance software and using acceleration information, the vehicle sequencing software generates the sequencing commands for staging events. The sequencing commands are routed through appropriate sequencing electronics.

The control commands generated by the autopilot are processed in stage processors and the appropriate control command is issued to each control power plant. The movement of the control power plant provides the necessary control forces and moments, which in turn orient the vehicle in the desired direction. In addition to actuating the control power plant, the upper stage control electronics processes propulsion control also.

Appropriate redundancy is built into the system, both at the sensor level and at the computer segment. The onboard computer segment is fault-tolerant with two chains working in parallel. These chains are configured as prime and redundant systems that operate synchronously. The subsystems communicate with each other through paired and cross-strapped links, so that the total system can tolerate non-identical multiple failures.

\subsection{Propulsion control}

Thrust levels and mixture ratios achieved in propulsion systems during flight are based on engine tuning parameters at ground. Due to the tuning inaccuracy and engine/propellant operating conditions, these parameters can widely vary for cryogenic engines. Hence, to ensure engine safety and optimum engine performance, optimum thrust level and defined mixture ratios need to be achieved. This have impact on the mission also. Optimum mission performance is achieved with the nominal acceleration profile (thrust profile). Also, if the nominal mixture ratio is ensured, then all the loaded propellant is utilized for the mission, thus resulting in better propellant management. For this purpose, thrust regulation and mixture ratio control are implemented in cryogenic engines. Using sensor data, propulsion control is carried out by control electronics of upper stages. The schematic of this system is given in figure 2.

For thrust regulation, accelerometer output of navigation system is used. For mixture ratio control, flowmeters for both fuel and oxidizer, and temperature measurement of oxidizer are used.

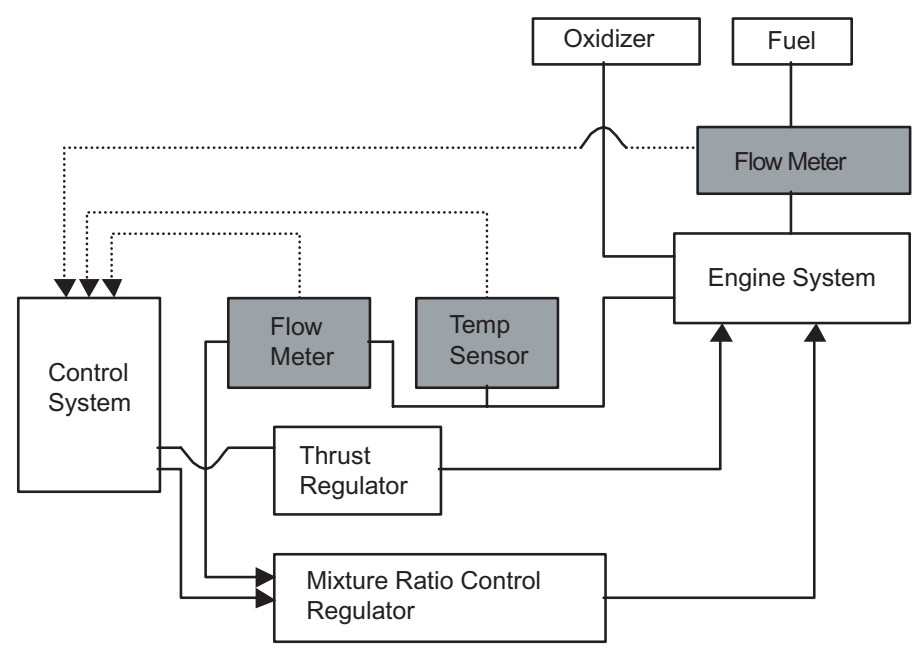

Figure 2. Propulsion control system. 
The thrust-regulation software regulates thrust to maintain predefined acceleration profiles. The thrust of the engine is varied by regulating the oxidizer flow into the gas generator. The mixture ratio control software computes the instantaneous mixture ratio of the engine and then computes the necessary control command to maintain the mixture ratio at the required level. Mixture ratio control is achieved by controlling the flow control valve on the oxidizer line at the inlet of the engine combustion chamber. In propulsion control also, total redundancy is built into the system, both at the sensor level and at the computer segment.

\section{Sensor data processing and performance requirements}

The mission performance and accuracy depend on the sensor performance. In the integrated NGC-PC system, the sensor management is as given below.

\subsection{Sensor management}

3.1a Multifunction sensors: In this type, the same sensor data is used for different functions. Accelerometer and gyros are multifunction sensors. The accelerometer data is used for navigation, sequencing and thrust regulation functions. The rate derived by gyros is used for navigation and autopilot functions.

3.1b Multi-sensor system: Different sensor outputs are used for a single function. The autopilot system uses rates from navigation gyros, and rate gyros and attitude computed by navigation.

3.1c Autonomous sensor system: In this type, the sensor outputs are used for a defined function. The flow-meters and temperature sensors are used for propulsion control.

Sensor characteristics and sensor accuracy requirements are different for different systems. For the case of multifunction sensors, the performance requirements are dictated by the mission accuracy and the subsystems requirements are met by the appropriate filtering of the same sensor data.

\subsection{NGC sensors}

Accelerometers and gyros mounted in the navigation system are the main sensors. In addition, rate gyro sensor outputs are used for control computations. The gyros and accelerometers are body mounted. The angular rates sensed by gyros include the rigid body vehicle rotation, flexible mode of the vehicle, flexible modes of deck plate, and slosh mode of the vehicle.

Similarly, the accelerometer senses the linear acceleration of the rigid body due to propulsion, aero dynamics and control systems, linear vibrations induced by the propulsion systems and linear acceleration due to rigid/flexible body rotations.

The zero frequency performance of the gyros is characterized by the error coefficients viz., fixed drift rate, $g$-sensitive drift rate, $g^{2}$-sensitive drift rate, aniso-inertia drift rate and scale factor variations. The accelerometer zero frequency performance is characterized by the error coefficients viz., bias, scale factor variations, and scale factor nonlinearity. These sensor coefficients have a mean value and day-to-day variation. By calibration and compensation of these error coefficients, the only uncertainty left is day-to-day variation. The short-term variation of error coefficients can be ignored for a short-duration mission. The mission performance depends mainly on uncompensated errors in these coefficients. The dynamic performance of the sensor is determined by the rebalance loop frequency response. 
The sensor data are derived in both digital and analog routes. For highly accurate computations, the digital route, which integrates out the sensor noises, is useful. The instantaneous requirement is met by analog route, but with less accuracy.

3.2a Navigation functions: Navigation functions use digital data of gyros and accelerometers. Error compensations are carried out on the velocity and angular increments. Since mission accuracy depends on the correctness of navigation data, gyro and accelerometer performance requirements are dictated by the navigation requirements.

The principal sources of errors affecting the navigation performance are gyro-related errors and accelerometer-related errors (day-to-day, uncompensated), initial alignment errors, and voltage to frequency conversion-related errors. The computational errors are caused by factors like algorithmic errors, simplified models for earth's gravity and use of finite word length. The permissible $3 \mathrm{~s}$ errors in navigation data due to the computational process are specified for position, velocity and computational drift. The permissible values for these parameters are specified and the system should ensure that the day-to-day stability errors meet these specifications. This will satisfy the ultimate mission dispersions specified.

3.2b Vehicle sequencing requirements: For vehicle sequencing, the burn-out of each stage is to be decided onboard. Since fall of acceleration indicates the start of tail off, acceleration data are used for vehicle sequencing. For this function, accuracy is not important, but the sharp fall in the tail off is to be captured. The analog data of acceleration are most suitable for this function. The analog acceleration data is passed through a low-pass filter and used for this purpose. The filter bandwidth is based on the upper and lower bound tail-off of characteristics.

3.2c Autopilot requirements: In addition to following the computed attitudes, the autopilot needs to ensure the stability of the control loop, and to avoid control-structure-slosh interactions. In order to function satisfactorily in the environment of vehicle flexible mode of different frequencies, vehicle slosh mode frequencies and bandwidths of control power plant actuators, control bandwidth of each stage and response of attitude rate sensors are specified.

Low-pass filters are included in the gyro channel to obtain the attitude with the specified bandwidth. Since the rotational dynamics are fast enough, the instantaneous rate information required should have high bandwidth. Hence analog data are used for this purpose. The required bandwidth is achieved through the low-pass filter in the analog channel of the gyro. During first-stage flight, in order to stabilize the vehicle flexible modes, in addition to the gyro rates in navigation system, the rates acquired by rate gyros located at inter stage is combined and the blended rate is used as vehicle attitude rates. For this purpose, necessary filters are used in rate gyros channels to get the required bandwidth.

$3.2 \mathrm{~d}$ Thrust regulation requirement: For regulating thrust, the propulsion control system requires acceleration. Since the propulsion system response is slow compared to that of the NGC system, the data are required only at larger intervals but with higher accuracy. Hence the digital data are used to compute velocity increments to achieve the required accuracy.

\subsection{Propulsion control sensors}

For mixture ratio control, flowmeters and temperature sensor data are used. The flowmeter data is very sensitive for mixture ratio control. Since the propulsion system response is slow, control needs to be carried out at slower rates. Instantaneous flowmeter data usage leads to 
instability because of sensor noise and sensor characteristics. Hence, an appropriate averaging scheme is used for flowmeter measurement.

Various factors which contribute to the inaccuracy in maintaining mixture ratio and thrust regulation are errors due to sensor inaccuracy, and acquisition of signals, computational errors, errors due to dead band, propellant temperature change, mass uncertainties including loading, and performance dispersion. From this, the performance requirements of propulsion control system with $3 \sigma$ dispersion are defined.

\section{Redundancy management of sensors}

In all mission critical sensors, hardware redundancies are built-in. The FDI logics are implemented to isolate failed and performance-deviated sensors. The usage of failed sensor output leads to mission failure, hence it should be isolated. Even without failure, sensor performance deviation beyond the specification leads to mission failure, hence the performance-deviated sensor should be isolated. Owing to limitations on the number of sensors, if further isolation is not possible, then decision is made to salvage the mission with predefined profiles. If none of the sensor outputs are isolated, then to achieve the best mission accuracy, the proper sensor output is selected using appropriate logics.

FDI logics are essential to achieve mission accuracy in the environment of failure of sensors, quantization errors, uncompensated errors due to uncertainties in sensor performance, systematic uncompensated software errors, and phase difference between the measurements.

\subsection{Thrust accelerometer FDI logic}

There are three accelerometers along the thrust axis of a vehicle. The requirements of the FDI logic for these sensors are:

- one accelerometer failure will be detected and isolated, making use of triple modular redundancy (TMR),

- failure of two accelerometers will be detected and isolated for mission salvage;

- the best accelerometer data will be used to achieve mission accuracy.

In the analog route, simple TMR logic is used for selecting the accelerometer data. The digital route data is used for navigation and thrust regulation purposes, wherein high accuracy is required. The FDI logic is implemented at three levels, viz.,

(a) to isolate the failed accelerometer using simple bound check;

(b) to isolate the performance degraded sensors using hard failure detection;

(c) to identify the best accelerometer data to achieve best mission accuracy using soft failure detection.

All the checks are done continuously during the entire flight duration. While the bound check is done on raw sensor data, the hard and soft failure checks are done on the compensated sensor outputs. While the bound checks and hard failure checks are done on instantaneous data, the soft checks are done on the accumulated data for well-defined numbers of cycles. If bound check and hard failure checks isolate two sensors, then the data selected in the previous cycle are used for next few cycles, which are defined. Beyond that, the mission salvage logic is used.

If all three sensors pass the bound check, then majority voting logic for hard failure detection is used. In majority logic, deviation of each sensor output with respect to the mean value 
is compared with predefined threshold values. If all three-sensor values pass this test, then majority logic is used for soft failure detection, wherein average of all three is used. Elaborate logics have been built for different cases of failures and tested extensively for the correctness of the logics. In case there is total failure, then mission salvage mode is exercised.

\subsection{Lateral accelerometer FDI}

For each lateral direction, only one sensor is used. Once the failure of lateral accelerometer is detected, zero value is used after isolating the faulty sensor. The same logic is used for both lateral accelerometers.

\subsection{FDI logics for gyros}

To provide redundancy, three two-axis gyros are used in skewed configuration. One of the sensor axes is aligned with a body axis and the other axis is in the orthogonal plane, which senses the rates about the remaining two axes. Because of this configuration, for the rates about each axis, there are three measurements available; one measurement is direct and other two are derived from the outputs of other sensors. Hence, parity equation residual checks are done for FDI of each axis. The requirements are:

- The FDI logic for gyros will detect and isolate the first failure of a Gyro, either single axis or both axes by parity equation residual checks.

- Wheel speed status will be considered when parity checks show an unidentifiable situation.

- A reinduction delay feature will be provided to reduce the frequent switching between gyro configurations.

For this purpose, the gyro FDI is organized at four levels by introducing appropriate logics to identify the failures. The same logics are used for both digital and analog routes of gyro outputs. These logics are tested extensively at different levels, including an environmental test, with necessary hardware and software for their evaluation.

\subsection{FDI logics on usage of acceleration data for thrust regulation}

Detailed FDI logic is implemented for accelerometer data as part of navigation functions. The selected value of accelerometer data along with accelerometer failure flag is used in the thrust regulation algorithm.

If the accelerometer fails, thrust regulation using accelerometer data is not possible, hence from the engine safety point of view; thrust regulation is carried out with predefined failsafe profile. Since, accelerometer FDI is already carried out, the correct data are used in the algorithm. Abnormal engine performance leads to very low or very high thrust, which in turn would reflect on the accelerometer data. Hence, bound check is done on the selected accelerometer data. If the bound check fails, it is assumed that the engine performance is abnormal and hence from the engine point of view, thrust regulation is done through predefined fail-safe profile.

\subsection{Flowmeter FDI}

At any instant, 3 numbers of flowmeters data are available for oxidizer and fuel. FDI logic implemented is to isolate the failed sensor data and to select the correct value. If two 
measurements match closer, then that is the most probable flow rate and hence median data are selected. If all three are widely varying, then it is difficult to assess and hence, from engine's safety point of view, mixture ratio control is done through predefined fail-safe profile. If the selected value is very high or very small, then engine function is assumed to be abnormal and regulation is through fail-safe profile.

\subsection{Temperature sensor FDI}

The resistance temperature detector is used as the temperature sensor. Three temperature sensor outputs are used. These values are processed and median value is used for computation.

\section{Performance evaluation of NGC-PC systems}

The performance evaluation of NGC and propulsion control elements are carried at development phases and extended up to the actual launch. After design phase, performance evaluations are carried out at subsystem level tests. From then onwards, the actual NGC-PC elements are added one by one at different levels of tests, till the maximum number of actual NGC-PC elements including sensors are brought into the loop and validated in the flight configuration under simulated flight environment. Different test beds with specific test objectives are used at different levels. Only after satisfactory performance, the NGC-PC system is cleared for flight integration. In this section, a brief summary of subsystem level tests and details of integrated NGC-PC system validation are given.

\subsection{Subsystem level tests}

Subsystem level tests are carried out on both software and hardware elements. For the software elements, application software, system software, interface requirements with other systems, data acquisition part, Digital to Analog and Analog to Digital converters etc., are tested. Generally, open loop tests are carried out using simulated input profile and the results are compared with reference output for validating system performance.

In the subsystem level tests of hardware elements, the sensors are mounted in the motion simulator and evaluated for their performance against the specification. If the performance is not satisfactory, appropriate corrective actions are taken to meet the required specifications. The gyros are mounted on angular motion simulator and accelerometers are mounted on the centrifuge to evaluate the sensor characteristics viz., systematic errors and stability values. The systematic errors observed in these tests are compensated during flight. Also, these tests provide realistic information on sensor dynamics and characteristics, which are useful to create the true mathematical model of the sensors hardware. These models are used extensively in further simulations wherein the realistic environments are not possible to simulate for the sensors.

\subsection{Integrated system level tests}

The objective of integrated system level test is to connect all the NGC-PC elements in the flight configuration and evaluate mission and subsystem performances in the simulated flight environment (Sivan et al 2000). A single test bed with all elements is not possible and realistic because of the following reasons:

- Interactions between different subsystems to be evaluated before connecting all the subsystems; 
- May not be possible to simulate realistic flight environment for all the subsystems in a single test bed;

- Because of sensitive hardware elements (sensors) are involved, the software elements may not be tested fully.

- All the subsystems may not be available for testing initially.

Hence, performance evaluations are carried out initially with lesser numbers of NGC-PC software elements and subsystem elements are added progressively. Finally, all the software and hardware elements are integrated and tested.

The final performance evaluations are carried out in hardware-in-loop simulation test bed (Chidambaram et al 1996, 2002; Sivan et al 2000). In this test, all the NGC-PC software and hardware elements including sensors are connected in flight configuration. The test bed configuration is given in figure 3. The flight sensors are mounted on an angular motion simulator (AMS), which simulates vehicle attitude motion to the sensors. The sensor outputs are interfaced with flight computers as in flight. Wherever it is not possible to use sensors, like flowmeters, appropriate simulators are used, that exactly simulate flowmeter behaviour. The loop is closed through a simulator and its features are:

- simulates the environment in which the vehicle flies ( atmosphere, wind, gravity etc.);

- simulates vehicle characteristics (aerodynamics, solid propulsion system, liquid engine model, cryo stage model, slosh, mass properties etc.);

- vehicle and trajectory dynamics (translational, rotational, elastic motion);

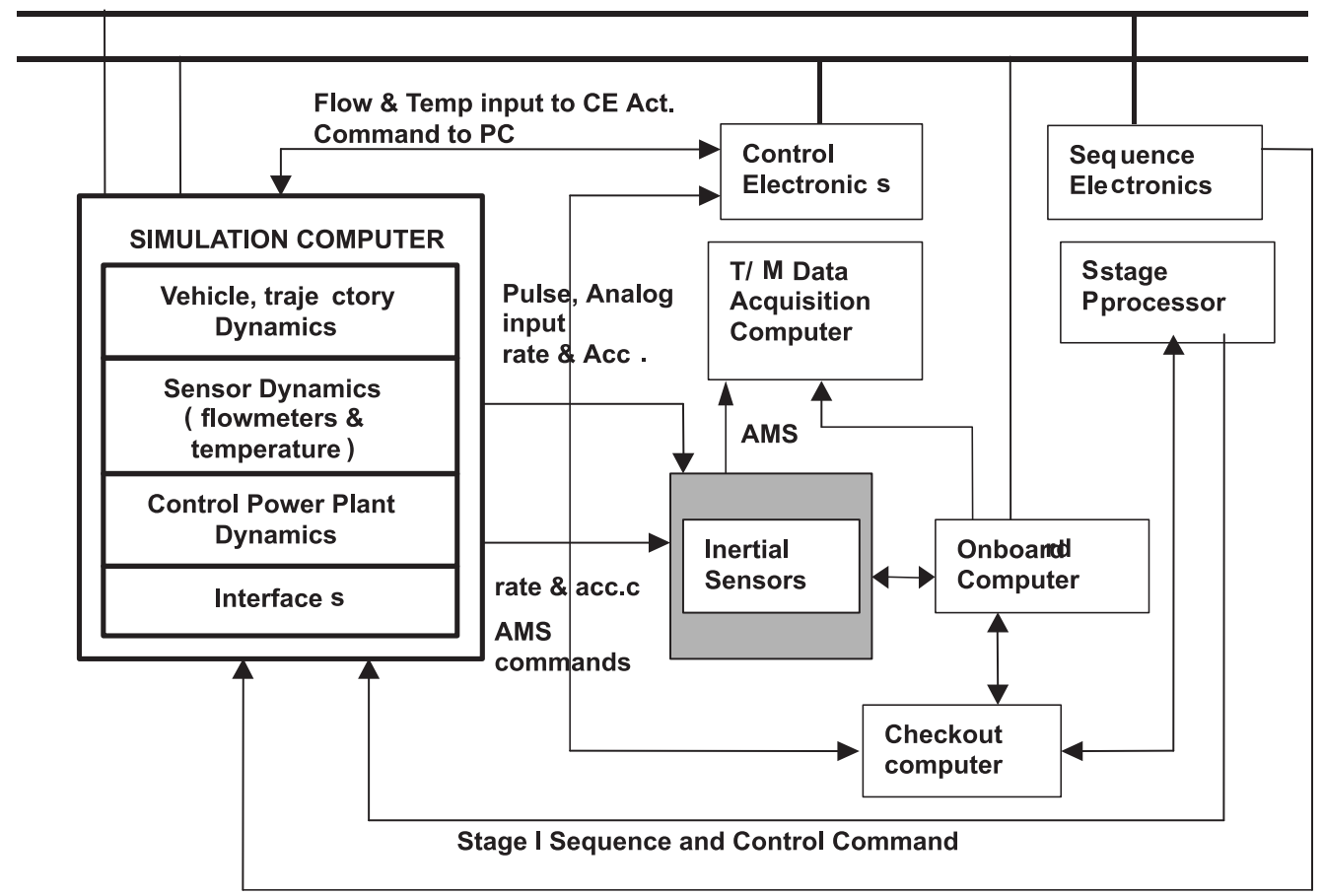

Stage II and Stage III Sequence and Control Command

Figure 3. Test bed configuration. 
- sensor dynamics of flow meters and temperature sensors;

- control power plant dynamics;

- interfaces with onboard system.

The simulation software commands the AMS to simulate vehicle motion to sensors and provides necessary information to onboard system as expected from a real vehicle. The simulation loop is closed with the vehicle attitude control commands, sequencing information and propulsion control commands as generated by onboard system.

The simulation software is synchronized with the onboard system. The simulation is started from flight initiation file loading through check out computer as in flight and is continued up to satellite injection. During simulation run, the telemetry data generated by onboard system is acquired in telemetry computer. Hence each simulation is equivalent to an actual flight with actual NGC-PC system and produces voluminous amount of information which contains vehicle parameters, trajectory data, mission details including achieved orbit, and telemetry data acquired and generated by other systems. From this information, the mission performance, subsystem performance, sensor performance etc, are evaluated. The hardware-in-loop-simulation (Puri 2002) is the ultimate test wherein maximum number of system hardware is brought into simulation loop. The main objectives of this simulation are:

- to evaluate the mission performance under the closed loop environments of NGC-PC elements including sensors for nominal and dispersed flight environments;

- to evaluate the effects of sensors errors on mission;

- to evaluate the mission performance under redundancy management;

- to evaluate NGC-PC software elements performance in the flight configuration;

- to evaluate the hardware pre filters effectiveness;

- end to end sign check.

\subsection{Typical simulation results}

The NGC-PC system undergoes very exhaustive simulations to evaluate its performance under all conditions of flight including failure modes. This section presents a few typical results of simulations to highlight their impact on the system and the corrective measures taken to make the system more robust.

In a typical example of a simulation with actual hardware, the simulator estimates the actual orbit achieved by the vehicle, whereas the onboard system computes orbit as estimated from the sensor data. The results of simulation with hardware were compared with the orbit achieved by the ideal sensor. In this simulation with actual hardware the achieved orbit varied significantly from run to run from the specified onboard orbit. From run to run, the actual orbit achieved became more and more eccentric. This was due to the sensor error increase from run to run.

On further analysis, it was confirmed that performance of one of the gyros was inconsistent and it was noticed that it happens only in flight configuration in the simulated flight motion. After modifications in the gyros, the simulations were repeated. The results were satisfactory and system was cleared for flight integration.

Results of hardware-in-loop-simulation for another typical launch mission with all sensors were as desired and there was repeatability. The orbit achieved in complete model simulation and simulation with all hardware elements was same. This confirmed the health of sensors and NGC-PC elements. 

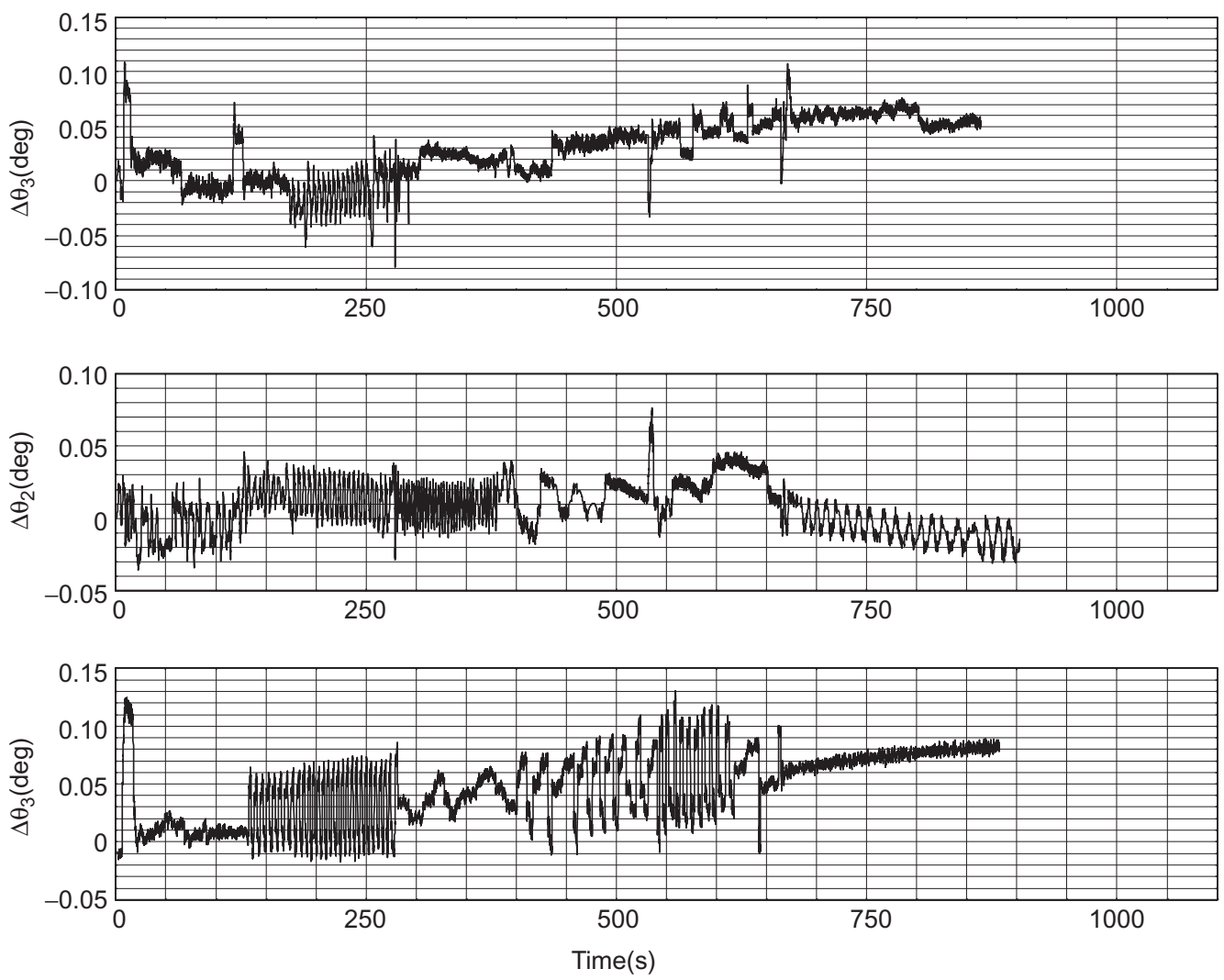

Figure 4. Attitude defference between actual and onboard estimation.

To evaluate the sensors performance, the difference between actual attitudes (three Euler angles)and attitudes estimated in onboard using sensor data are compared and shown in figure 4 . This also confirms that the sensor errors are almost zero.

In one of the simulations for yet another launch vehicle mission design, with initial version of the NGC-PC systems, limit cycle oscillation was observed in thrust regulation angles and achieved thrust as seen in figure 5 .

On further analysis, it was observed that there was a delay introduced in the propulsion control command generation. This was identified only in the integrated simulation with all hardware and software elements. After necessary modifications, the system performance was normal.

\section{Conclusions}

Multi-sensors with different types and characteristics are required in launch vehicles to achieve precisely the mission-defined orbit. To achieve the required mission accuracy, the sensors performance parameters should necessarily be within the specified bands. In a mission critical system, built-in sensor redundancy management along with FDI logics is needed to detect and isolate the failed or performance degraded sensors. An integrated system level test bed is 

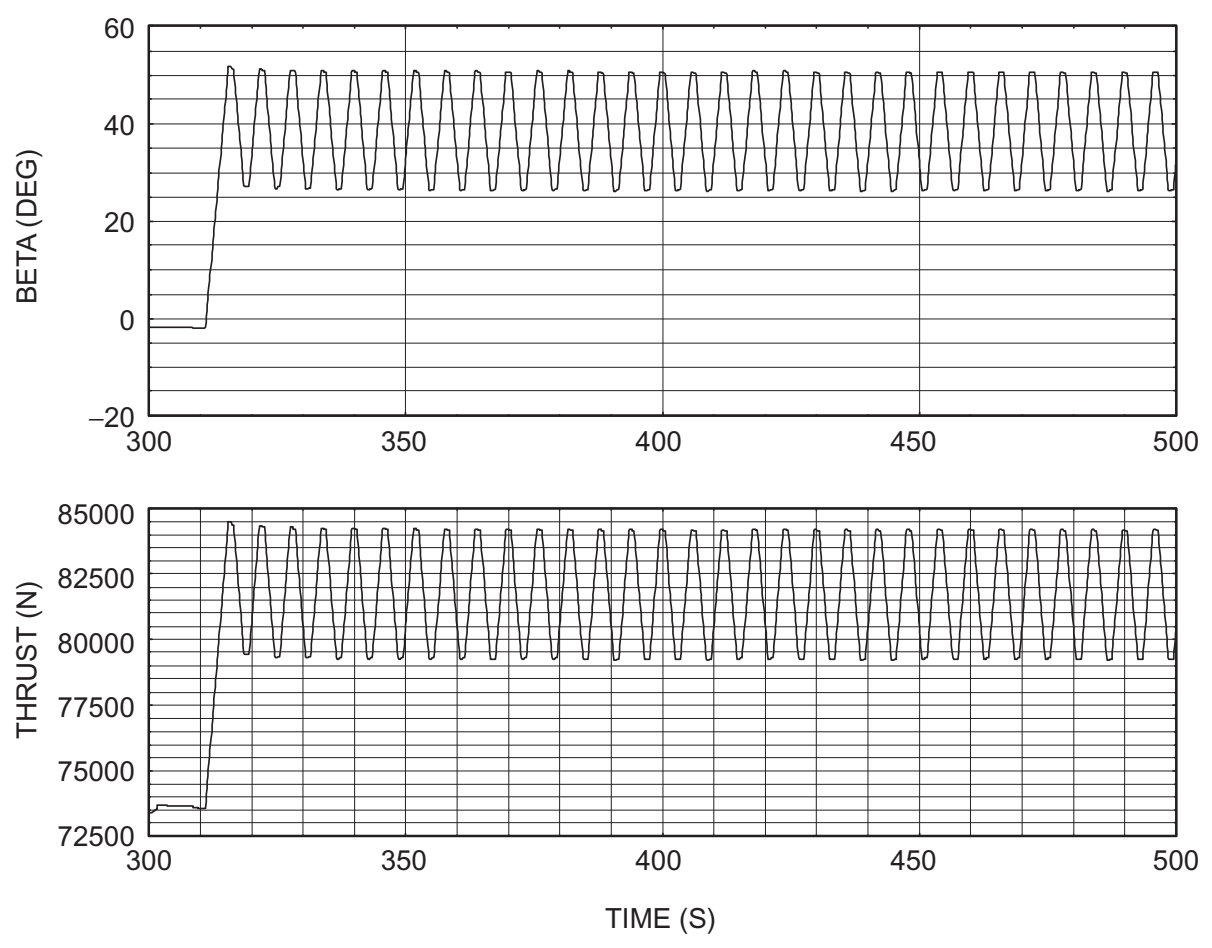

Figure 5. Thrust regulation angle and thrust.

required to evaluate the sensor and mission performance in the simulated flight environment. Some of the typical simulation results observed during launch vehicle mission simulation establishe the effectiveness of integrated system level test.

\section{References}

Chidambaram T R, Sivan K, Sudar I, Sreelatha A 1996a Integrated flight software validation for launch vehicle mission. Proc. National Workshop on Flight Software (Thiruvananthapuram: Systems Society of India) pp. 4.1-4.14

Chidambaram T R, Sivan K, Lal V B, Sudar I, Sreelatha A 1996b Hardware-in-loop simulations for testing inertial guidance system elements of launch vehicle. J. Aerosp. Soc. India 48: 331-337

Chidambaram T R, Sivan K, Sudar I 2002 Integrated testing of inertial navigation systems of launch vehicle. Proc. National Workshop on Navigation Systems - Present scenario and future trends (Thiruvananthapuram: Systems Society of India)

Gupta A K, Sajeendran 2002 Test and calibration systems for inertial sensors. Proc. National Workshop on Navigation Systems - Present scenario and future trends (Thiruvananthapuram: Systems Society of India)

Puri S N 2002 Navigation system development and its performance for GSLV-D1 mission. Proc. National Workshop on Navigation Systems - Present scenario and future trends (Thiruvananthapuram: Systems Society of India)

Shukkoor A A, Bhattacharyya K C, Gopakumar S, Hemachandran S, Santha Kumari K K, Syamala S 2000 Simulation testing of strapdown inertial navigation system and software. Proc. National Workshop on Aerospace Flight Simulation (Thiruvananthapuram: Systems Society of India) 
Sivan K, Sreelatha A, Chidambaram T R 2000a Integrated validation of navigation, guidance and control systems of launch vehicles through simulations. Proc. National Workshop on Aerospace Flight Simulation (Thiruvananthapuram: Systems Society of India)

Sivan K, Lal V B, Sudar I, Chidambaram T R 2000b Simulation facilities for validating navigation, guidance and control system of launch vehicles. Proc. National Workshop on Aerospace Flight Simulation (Thiruvananthapuram: Systems Society of India)

Sivan K, Sudar I, Sreelatha A, Chidambaram T R 2000c Actuator-in-loop simulation for validating satellite launch vehicle navigation, guidance and control systems. Proc. 24th National Systems Conference, Bangalore, pp. 62-73 